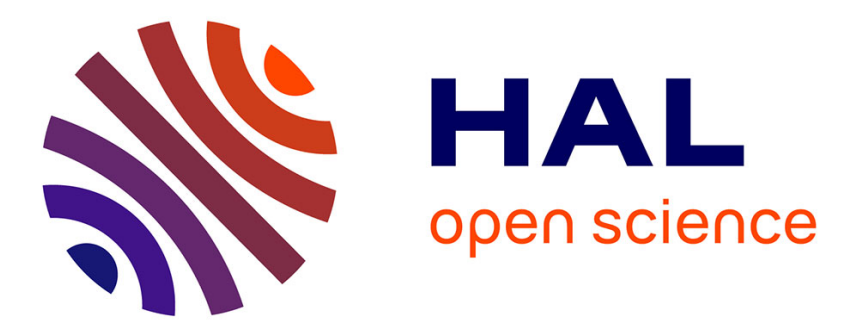

\title{
A Compact Representation of Random Phase and Gaussian Textures
}

\author{
Agnès Desolneux, Lionel Moisan, Samuel Ronsin
}

\section{To cite this version:}

Agnès Desolneux, Lionel Moisan, Samuel Ronsin. A Compact Representation of Random Phase and Gaussian Textures. IEEE International Conference on Acoustics, Speech, and Signal Processing (ICASSP), Mar 2012, Kyoto, Japan. pp.1381-1384, 10.1109/ICASSP.2012.6288148 . hal-00662377

\section{HAL Id: hal-00662377 https://hal.science/hal-00662377}

Submitted on 23 Jan 2012

HAL is a multi-disciplinary open access archive for the deposit and dissemination of scientific research documents, whether they are published or not. The documents may come from teaching and research institutions in France or abroad, or from public or private research centers.
L'archive ouverte pluridisciplinaire HAL, est destinée au dépôt et à la diffusion de documents scientifiques de niveau recherche, publiés ou non, émanant des établissements d'enseignement et de recherche français ou étrangers, des laboratoires publics ou privés. 


\title{
A COMPACT REPRESENTATION OF RANDOM PHASE AND GAUSSIAN TEXTURES
}

\author{
Agnès Desolneux, Lionel Moisan and Samuel Ronsin \\ Université Paris Descartes, MAP5, CNRS UMR 8145, Paris, France
}

\begin{abstract}
In this paper, we are interested in the mathematical analysis of the micro-textures that have the property to be perceptually invariant under the randomization of the phases of their Fourier Transform. We propose a compact representation of these textures by considering a special instance of them: the one that has identically null phases, and we call it "texton". We show that this texton has many interesting properties, and in particular it is concentrated around the spatial origin. It appears to be a simple and useful tool for texture analysis and texture synthesis, and its definition can be extended to the case of color micro-textures.
\end{abstract}

Index Terms - random phase texture, Gaussian random field, texture synthesis, texture analysis

\section{INTRODUCTION}

Texture images may have very different perceptual or statistical properties, and we will choose here to follow the discussion on textures as it was proposed in the paper of Galerne, Gousseau and Morel [1]. From their viewpoint, the set of all textures can roughly be divided into two classes: the macrotextures and the micro-textures. The macro-textures can be described as images containing visual objects spatially organized in a regular way (like a brick wall for instance). On the contrary, micro-textures do not contain well-identified visual objects, and they are characterized by the property of being perceptually invariant under the randomization of the phases of their Fourier Transform. This is not the case of macrotextures because changing the phases completely destroys the "objects" present in the image. Among all possible values for the phase field, we will study here a particular one: the identically null phase field. We will show that this simple choice has many interesting properties both for the analysis and for the synthesis of textures. The image obtained by setting to zero all the phases of an input micro-texture will be called the texton of the micro-texture. We will see that this image is a compact representation of the texture and that it moreover has the two fundamental characteristics of textures described by Julesz ([2], [3]): it is related to the second-order statistics of the texture, and it has an elementary shape that characterizes

This work has been supported by the French National Research Agency under grant ANR-09-BLAN-0029-01. the texture. This is the reason why we use the word texton introduced by Julesz.

\section{DEFINITION AND PROPERTIES}

The images we will consider are defined on a discrete rectangular domain $\Omega$ of size $M \times N$, that is given by $\Omega=I_{M} \times I_{N}$ where for $n$ integer, $I_{n}$ is the discrete interval $\left[-\frac{n-1}{2}, \ldots, \frac{n-1}{2}\right] \cap \mathbb{Z}$ when $n$ is odd, and $\left[-\frac{n}{2}, \ldots, \frac{n}{2}-1\right] \cap \mathbb{Z}$ when $n$ is even. For a discrete grey-level image $u: \Omega \rightarrow \mathbb{R}$, we will denote by $\widehat{u}$ its discrete Fourier Transform. The image $u$ can also be extended by periodicity to a function defined on $\mathbb{Z}^{2}$ by setting for all $y \in \mathbb{Z}^{2}, u(y)=u\left(y_{1}, y_{2}\right)=$ $u\left(y_{1}(\bmod M), y_{2}(\bmod N)\right)$. In particular, this allows us to define the (periodic) translation of an image $u$ by a vector $y$, denoted by $\tau_{y}(u)()=.u(y+$.$) . In the sequel, we$ will also use the following notations: if $x=\left(x_{1}, x_{2}\right)$ and $\xi=\left(\xi_{1}, \xi_{2}\right)$ are two points in $\Omega$, we will define and denote their discrete inner product by $\langle x, \xi\rangle=\frac{1}{M} x_{1} \xi_{1}+\frac{1}{N} x_{2} \xi_{2}$, so that the discrete Fourier Transform of $u$ is simply given by $\widehat{u}(\xi)=\sum_{x \in \Omega} u(x) e^{-2 i \pi\langle x, \xi\rangle}$.

We now introduce the texton of an image $u$ by the following

Definition 1 (Texton of an image). The texton of an image $u: \Omega \rightarrow \mathbb{R}$ is the unique image $T(u): \Omega \rightarrow \mathbb{R}$ that has the same Fourier amplitude as $u$, the same mean value as $u$ and that has identically null phases (except maybe in 0 ). In other words, the texton $T(u)$ is defined in the Fourier domain by

$\forall \xi \in \Omega, \xi \neq 0, \quad \widehat{T(u)}(\xi)=|\widehat{u}(\xi)| \quad$ and $\quad \widehat{T(u)}(0)=\widehat{u}(0)$

and, in an equivalent way, in the spatial domain by

$\forall x \in \Omega, T(u)(x)=\frac{1}{M N} \widehat{u}(0)+\frac{1}{M N} \sum_{\xi \in \Omega, \xi \neq 0}|\widehat{u}(\xi)| e^{2 i \pi\langle x, \xi\rangle}$.

\subsection{Elementary properties}

The texton $T(u)$ of an image $u$ satisfies many properties. In particular:

1. For any image $u, T(u)$ is a real and symmetric image, that is $T(u)(-x)=T(u)(x) \in \mathbb{R}$ for all $x \in \Omega$. 
2. $T(T(u))=T(u)$, meaning that $T(u)$ is its own texton.

3. The operator $T$ is 1-Lipschitz for the $L^{2}$ norm: if $u$ and $v$ are two images defined on $\Omega$, then

$$
\|T(u)-T(v)\|_{2} \leq\|u-v\|_{2} .
$$

4. The texton is translation invariant: $T\left(\tau_{y}(u)\right)=T(u)$ for all $y \in \Omega$.

5. For any image $u$ on $\Omega$, and for any real numbers $\alpha$ and $\beta$, we have

$$
T(\alpha u+\beta)=|\alpha| T(u)+(\alpha-|\alpha|) \bar{u}+\beta,
$$

where $\bar{u}$ is the mean value of $u$ on $\Omega$.

6. For any images $u$ and $k$ defined on $\Omega, T(k * u)=$ $T(k) * T(u)$, where $*$ is the periodic convolution operator on $\Omega$. In particular, if $k$ is a symmetric convolution kernel having a positive discrete Fourier Transform (a Gaussian kernel for instance), then $T(k * u)=k * T(u)$.

\subsection{Properties of concentration}

The texton $T(u)$ also satisfies some properties of spatial concentration around 0 . This can be clearly observed on texture images, and it can be mathematically explained by the following results. The first result is that if $u$ is an image defined on $\Omega$ that has a positive mean value, then $T(u)$ is the unique solution of the following problem of optimization: find

$v: \Omega \rightarrow \mathbb{R}$ that maximizes $v(0)$ under the constraint $|\widehat{v}|=|\widehat{u}|$.

Moreover, by the triangular inequality, the texton $T(u)$ reaches its maximum value in $x=0$. It can also be shown that the texton $T(u)$ is, among all the images having the same Fourier amplitude as $u$, the one that optimizes any even-order derivative at 0 . Such a derivative is given by

$$
\frac{\partial^{2 m+2 n} T(u)}{\partial^{2 m} x_{1} \partial^{2 n} x_{2}}(0)=\frac{\left(-4 \pi^{2}\right)^{m+n}}{M^{2 m+1} N^{2 n+1}} \sum_{\xi \in \Omega} \xi_{1}^{2 m} \xi_{2}^{2 n}|\widehat{u}(\xi)| .
$$

Notice that all odd-order derivatives are equal to zero at 0 because of the symmetry of the texton.

The second mathematical result is about the regularity of the Fourier transform of the texton. Indeed, it can be shown that if $u$ is an image with positive mean value (if this assumption is not satisfied, just change $u$ into $-u$ ), the texton $T(u)$ minimizes, among all images $v$ having the same Fourier amplitude as $u$, any $L^{p}$-norm $(p>0)$ of the discrete (periodic) gradient of the Fourier Transform, that is given by

$$
\begin{aligned}
\|\nabla \widehat{v}\|_{p}^{p}= & \sum_{\xi=\left(\xi_{1}, \xi_{2}\right) \in \Omega}\left(\left|\widehat{v}\left(\xi_{1}+1, \xi_{2}\right)-\widehat{v}\left(\xi_{1}, \xi_{2}\right)\right|^{p}\right. \\
& \left.+\left|\widehat{v}\left(\xi_{1}, \xi_{2}+1\right)-\widehat{v}\left(\xi_{1}, \xi_{2}\right)\right|^{p}\right) .
\end{aligned}
$$

In particular, when $p=2$, one can go back to the spatial domain by Parseval Theorem, and this then implies that the texton minimizes, under the constraint $|\widehat{v}|=|\widehat{u}|$, the following functional (which can be seen as a measure of spatial concentration around 0 ):

$$
E(v)=\sum_{\left(x_{1}, x_{2}\right) \in \Omega}\left(\sin ^{2} \frac{\pi}{M} x_{1}+\sin ^{2} \frac{\pi}{N} x_{2}\right) v\left(x_{1}, x_{2}\right)^{2} .
$$

\subsection{Gaussian textures}

The set of Gaussian textures is a large class of the set of all micro-textures. Among them, the periodic stationary Gaussian textures are particularly well described in the Fourier domain. Indeed, they are characterized by the following law: the coefficients of the Fourier Transform on a half-domain $\Omega_{+}$ are independent complex Gaussian random variables. This is equivalent to say that, on $\Omega_{+}$, the phases are i.i.d. uniform on $[0,2 \pi)$ and the amplitudes are independent following Rayleigh distributions. These Gaussian textures can be seen as the asymptotic limit of the so-called spot noise models of textures (introduced by van Wijk [4]). In the sequel, they will be denoted by ADSN (Asymptotic Discrete Spot Noise, following the term used in [1]). An ADSN texture can be written as $U=h * W$, where $h$ is an image with mean value zero, and $W$ is a white noise. The texton of $U$ is then given by $T(U)=T(h) * T(W)$. The analysis of the texton of such a texture is thus equivalent to the computation of the texton of a white noise image. Such a computation can be explicitly performed, and one can show that $T(W)$ is asymptotically "close" to a Dirac mass located in 0. More precisely, we have

Proposition 1. Let $W$ be a white noise image, that is, the $W(x)$ are i.i.d. following a reduced centered Gaussian distribution). Then, $T(W)$ is a random image that has the following first and second moments:

$$
\begin{aligned}
& \text { - } \mathbb{E}(T(W)(0))_{M N \rightarrow+\infty}^{\sim} \frac{\sqrt{\pi}}{2} \sqrt{M N} \\
& \text { and } \mathbb{E}(T(W)(x))=-\frac{\sqrt{\pi}}{2} \frac{1}{\sqrt{M N}} \text { for } x \in \Omega, x \neq 0 . \\
& \text { - } \operatorname{Var}(T(W)(0)) \underset{M N \rightarrow+\infty}{\sim} \frac{4-\pi}{2} \\
& \\
& \text { and } \operatorname{Var}(T(W)(x)) \underset{M N \rightarrow+\infty}{\sim} \frac{4-\pi}{4} \text { for } x \in \Omega, x \neq 0 . \\
& \text { - } \operatorname{Cov}(T(W)(x), T(W)(y))=\frac{\pi-2}{M N} \text { for } x \neq y
\end{aligned}
$$

This shows in which sense we can say that $T(W)$ is asymptotically close to a Dirac mass located in 0 when $N M \rightarrow+\infty$.

\section{APPLICATIONS}

\subsection{Texture Analysis}

A first application of the texton is for the purpose of texture analysis. Indeed, the texton is a "compact summary" of the properties of the texture: characteristic size, anisotropy, 
etc. This comes from the fact that, thanks to the product/convolution property of the Fourier Transform, we have that $C_{u}=\frac{1}{M N} T(u) * T(u)$ where $C_{u}$ is the empirical covariance of the image (assumed here to have a zero mean-value). As a consequence of this, the texton is much less blurry than the covariance (because this latter is obtained by an auto-convolution of the texton), and it permits to describe the second-order statistics of the texture in the same space as the texture itself (that is, without using square intensities). These properties make the texton particularly interesting for the statistical analysis of textures. This is illustrated on Fig. 1 , where we compare the texton and the covariance of a medical image (calcaneum bone, from the database used in [5] in relation to osteoporosis).
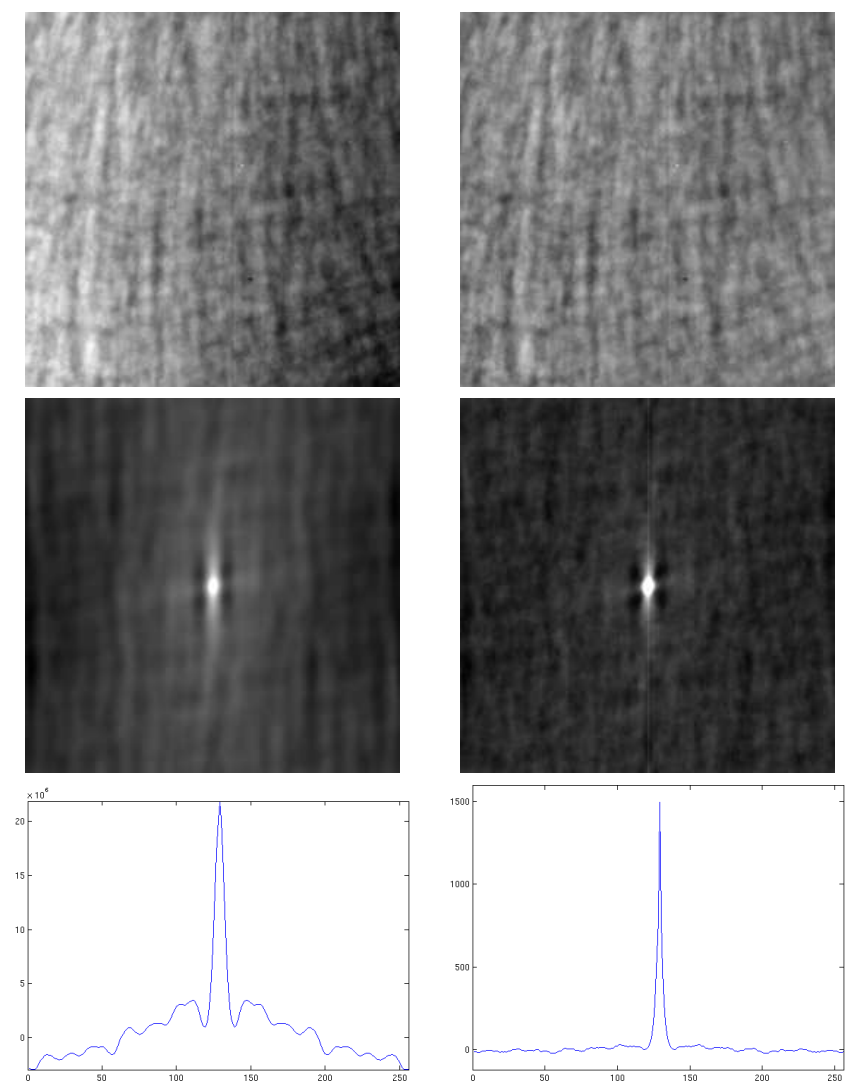

Fig. 1. Top: left, the original image (calcaneum bone) and on the right, its periodic component obtained by the periodic + smooth decomposition [6]. Middle: left, the empirical periodic covariance of this image, and on the right, the texton. This latter is clearly less blurry and more concentrated than the covariance. Bottom: intensities along the middle horizontal line $(y=0)$ of the covariance image (on the left) and of the texton image (on the right).

\subsection{Texture synthesis}

A second application of the texton is for the purpose of texture synthesis. We assume here again that the image has mean value zero (this can always be achieved by subtracting the mean intensity value). We have seen that the texton is very concentrated around 0 and it has very few high intensity values far from the origin. Consequently, we can perform a hard-thresholding of the texton and thus obtain a very near image (in the $L^{2}$ sense) that has a compact support. To synthesize a texture of any size, we then simply extend by the value 0 this compactly-supported texton approximation, and then randomize the phases of the Fourier Transform. This is illustrated on Fig. 2.
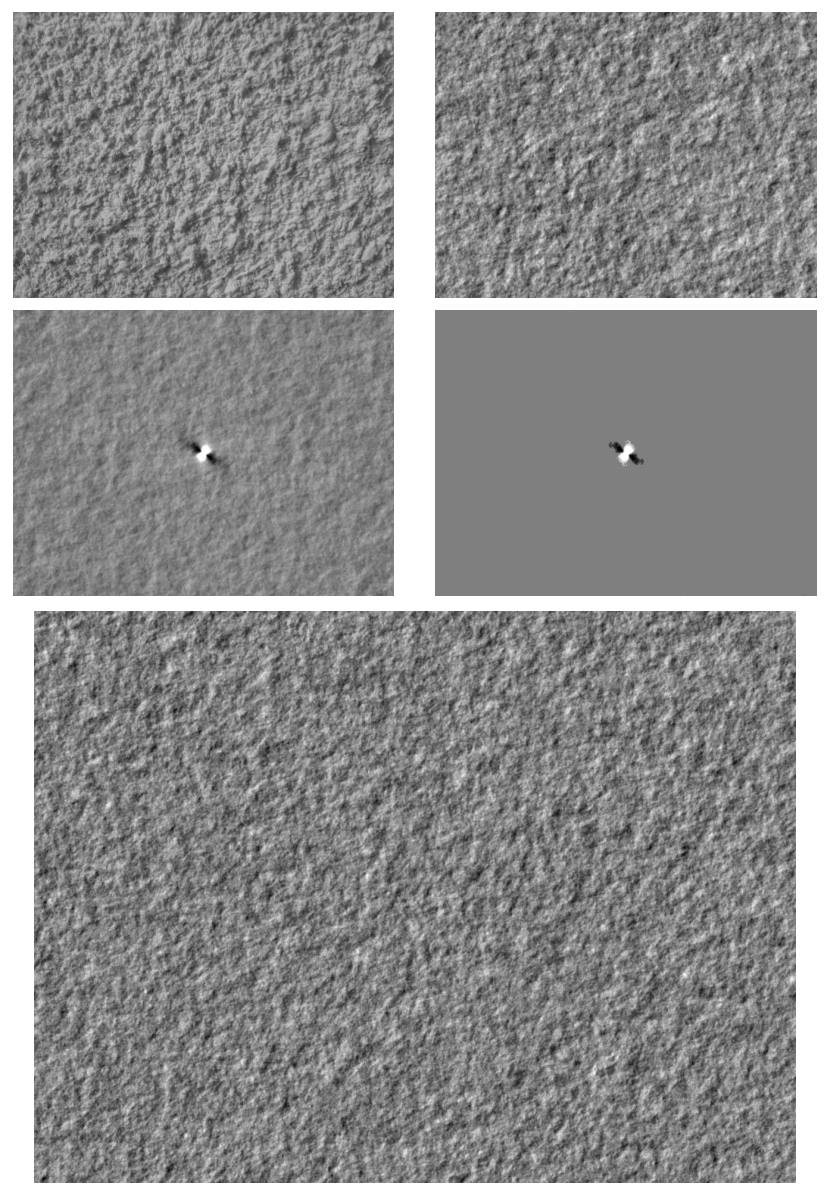

Fig. 2. Top left: the original texture image $u$ (some wall rendering). Top right: a sample of the phase randomization performed on $u$. Middle left: the texton $T(u)$ of the image $u$. It is clearly concentrated around 0 . Middle right: the texton $T(u)$ after a hard-thresholding. We obtain a compactly supported image that can be extended by 0 outside the image domain. It then allows us to synthesize, by phase randomization, texture images of any size. Bottom: a sample whose size is the double of the original texture image. 


\subsection{The case of color textures}

Let us also mention that the concept of texton can be extended to color textures. In that case, to define the texton properly (that is without creating any color artifact), we must not put an identically null phase for the three channels, but we must instead subtract the same phase (the one of the luminance for instance) to all of them. This has to be done in order to preserve the phase difference between the three channels. It can indeed be shown that this definition is the only possible one if we want to preserve the spectrum of all colors. More precisely, we have the following

Proposition 2. Let $\mathbf{u}=\left(u_{r}, u_{g}, u_{b}\right)$ be a color image defined on $\Omega$ and with values in $\mathbb{R}^{3}$. For any $\lambda=\left(\lambda_{r}, \lambda_{g}, \lambda_{b}\right) \in \mathbb{R}^{3}$, we denote by $\lambda \cdot \mathbf{u}$ the real-valued image defined on $\Omega$ by $\lambda \cdot \mathbf{u}(x)=\lambda_{r} u_{r}(x)+\lambda_{g} u_{g}(x)+\lambda_{b} u_{b}(x)$.

1. If $\mathbf{T}(\mathbf{u})$ is a color texton associated to $\mathbf{u}$, then the condition of the conservation of all Fourier amplitudes, that can be written

$$
\forall \lambda \in \mathbb{R}^{3}, \forall \xi \in \Omega, \quad|\lambda \widehat{\cdot \mathbf{T}(\mathbf{u})}(\xi)|=|\widehat{\lambda \cdot \mathbf{u}}(\xi)|,
$$

is satisfied if and only if there exists a phase field $\phi$ defined on $\Omega$, satisfying $\phi(-\xi)=-\phi(\xi)$ for all $\xi \in \Omega$, and such that

$$
\forall k \in\{r, g, b\}, \quad \widehat{\mathbf{T}(\mathbf{u})_{k}}(\xi)=\widehat{u_{k}}(\xi) e^{-i \phi(\xi)} .
$$

2. Let $\alpha=\left(\alpha_{r}, \alpha_{g}, \alpha_{b}\right) \in \mathbb{R}^{3}$, and let $\phi_{\alpha}$ be the phase field of the Fourier Transform of $\alpha \cdot \mathbf{u}$. Then, if we denote by $T_{\alpha}(\mathbf{u})$ the color texton of $u$ obtained by subtracting the phase $\phi_{\alpha}$ to the three channels (that means by taking $\phi=\phi_{\alpha}$ in Equation (4)), we get

$$
T(\alpha \cdot \mathbf{u})=\alpha \cdot T_{\alpha}(\mathbf{u}),
$$

where $T(\alpha \cdot \mathbf{u})$ is the texton of the real-valued image $\alpha \cdot \mathbf{u}$, as defined by Definition 1 .

An example of the color texton obtained from a color image, using its standard luminance (that is, $\alpha=(1 / 3,1 / 2,1 / 6)$ ) is shown on Fig. 3.

\section{REFERENCES}

[1] B. Galerne, Y. Gousseau, and J.-M. Morel, "Random Phase Textures: Theory and Synthesis," IEEE Trans. on Image Processing, vol. 20, no. 1, pp. 257-267, 2011.

[2] B. Julesz, "Visual pattern discrimination," IRE transactions on Information Theory, vol. 8, no. 2, pp. 84-92, February 1962.

[3] B. Julesz, "A theory of preattentive texture discrimination based on first-order statistics of textons," Biological Cybernetics, vol. 41, pp. 131-181, 1981.
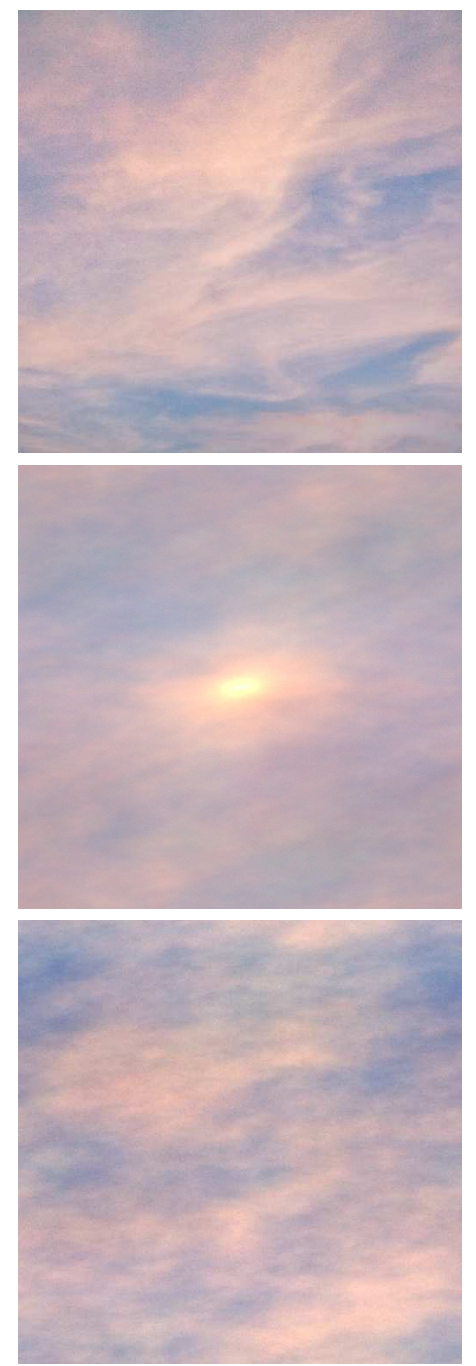

Fig. 3. Top: the original color image (it is the periodic component ([6]) of an image of a sunset). Middle: the color texton of this image obtained by subtracting the phase of the luminance to the three channels. Bottom: a sample of the phase randomization procedure (the same random phase is added to the three channels in order to preserve the phase differences, see Proposition 2).

[4] J. J. van Wijk, "Spot noise texture synthesis for data visualization," in SIGGRAPH '91, 1991, pp. 309-318.

[5] Gadois C. Kousignian I. Neveu J.P. Fardellone P. Kolta S. Roux C. Do-Huu J.P. Lespessailles, E. and C.L. Benhamou, "Clinical interest of bone texture analysis in osteoporosis: a case control multicenter study," Osteoporos Int., vol. 19, pp. 1019-1028, 2008.

[6] L. Moisan, "Periodic plus smooth image decomposition," Journal of Mathematical Imaging and Vision, vol. 39, no. 2, pp. 161-179, 2011. 